\title{
A case of isolated primary chylopericardium
}

\author{
PETER ROSS, SIMON JOSEPH, AND DUNCAN WALKER \\ From the Department of Cardiology and Cardiothoracic Surgery, The Middlesex Hospital, London
}

SUMMARY Primary chylopericardium presented as radiographic cardiomegaly in an asymptomatic 19-year-old man. Normal findings at cardiac catheterisation and angiographic demonstration of cardiac displacement from the diaphragm suggested a collection of fluid below the heart which was confirmed by $M$ and $B$ mode ultrasound scanning. Thoracic blood pool isotope scanning indicated that the lumen was a pericardial effusion rather than a cyst. Lymphangiography did not indicate any direct lymphatic communication though a small pool of contrast appeared in the pericardium and the diagnosis of chylopericardium was confirmed by pericardiocentesis.

Surgical treatment was undertaken after rapid reaccumulation of chyle and the patient remains well 6 months later.

\section{Case report}

An asymptomatic 19-year-old male army recruit was referred for investigation of an enlarged heart shadow noted on routine chest radiography. There was no relevant medical history apart from an accident at the age of 10 when a fall from a trampoline caused painful hyperextension of the back; this was not treated medically. Physical examination was normal. In particular, on palpation of the cardiac apex, the jugular venous pressure and auscultation were normal. The arterial blood pressure was $120 / 70 \mathrm{mmHg}$ without paradox.

A chest radiograph showed cardiomegaly (cardiothoracic ratio 0.53 ), with pyriform outline and the lung fields were clear. The lateral radiograph showed a diminished anteroposterior diameter and a 'straight back'. A curved shadow lying within the mediastinal border was thought to represent the apex of the left ventricle and was visible in both posteroanterior and lateral films (Fig. 1). Electrocardiography was normal, with a mean frontal plane QRS axis of $+90^{\circ}$. M mode echocardiography, with the transducer conventionally placed at the fourth left intercostal space just lateral to the sternum, failed to show any abnormality in spite of caudal direction of the ultrasound beam. Cardiac catheterisation was, therefore, undertaken and showed normal right and left-sided pressures. The right and left ventriculograms were normal, but the heart was separated from the left hemidiaphragm by an unopacified area of similar radiographic density to the cardiac shadow. In view of this appearance $M$ mode echocardiography was repeated with the transducer positioned in the sixth left intercostal space near the midclavicular line. There was an echo-free space inferior to the left ventricular myocardium, which was compatible with a pericardial effusion or cyst (Fig. 2).

Contact grey scale B scan ultrasound 1 images with the patient supine were recorded from several positions on the lower anterior chest wall and left upper abdominal quadrant. These showed a thin-walled echo-free space bounded superiorly by the heart and inferiorly by the left hemidiaphragm. It extended anteriorly to the chest wall and posteriorly to the lung (Fig. 3). The technique later proved to be a valuable aid to pericardiocentesis. A thoracic blood pool isotope scan after intravenous injection of $3 \mathrm{mCi}$ Technetium $99 \mathrm{~m}$-labelled albumin showed abnormal imaging of the heart, with a 'halo' surrounding the cardiac silhouette typical of pericardial effusion (Staab and Patton, 1973) (Fig. 4).

Lymphangiography with injection of $7 \mathrm{ml}$ 'Lipiodol' into each foot showed normal abdominal lymphatics. The thoracic duct was not visualised. A small pool of contrast which collected later in the pericardium was seen in the erect position and moved with gravity. The site of communication between lymphatic channels and the pericardial sac was not demonstrated.

Percutaneous pericardiocentesis yielded $750 \mathrm{ml}$ fluid which had the appearance and chemical

${ }^{1}$ Nuclear Enterprises Diasonograph NE 4102 with NE 4804 Grey-Scale attachment. 

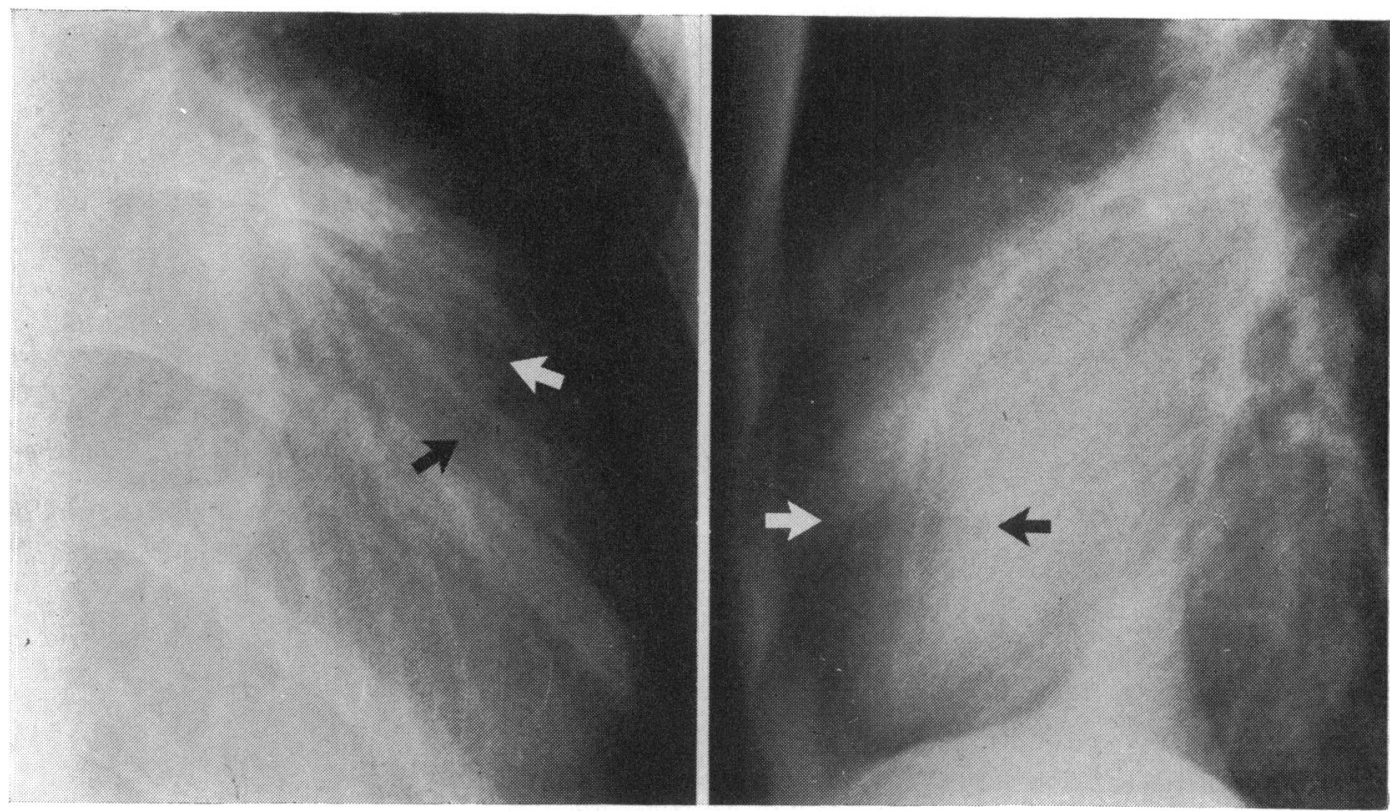

Fig. 1 Chest radiograph at presentation showing curved border of the left ventricle within the pericardial effusion.

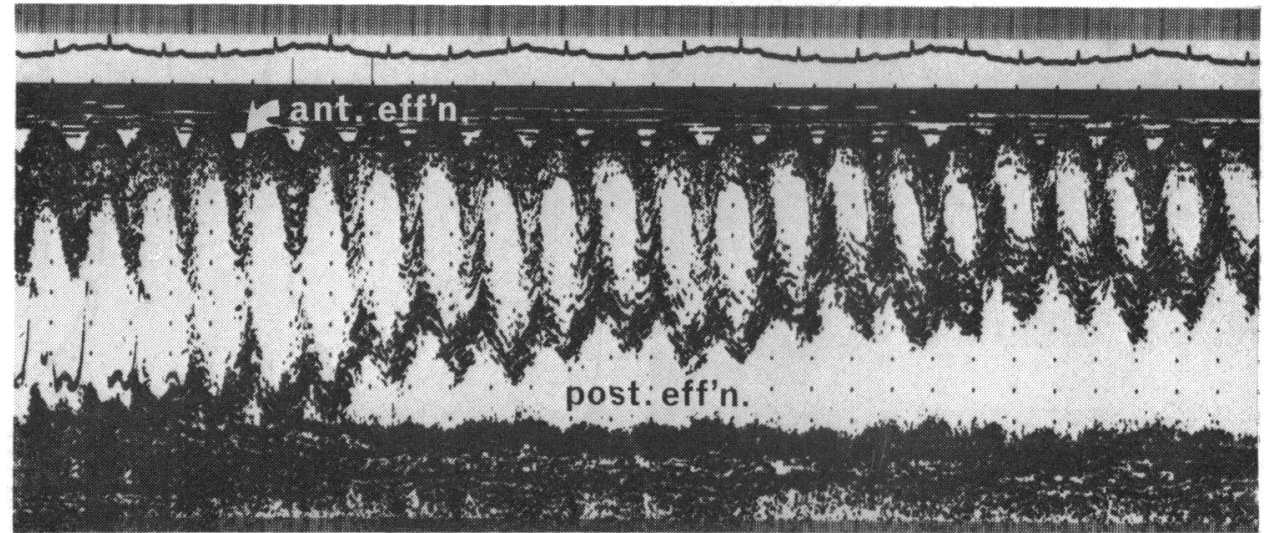

Fig. $2 M$ mode echocardiographic scan recorded with the transducer in the sixth left intercostal space and showing a small anterior echo-free space and a large posterior echo-free space.

characteristics of chyle (Table). Chest radiography one hour after aspiration showed a cardiothoracic ratio of 0.40 . There was a small amount of air in the upper pericardial sac. The electrocardiogram remained unchanged. Chest radiograph repeated after 72 hours showed reaccumulation of fluid. The patient remained asymptomatic with normal physical findings.

At thoracotomy an excess of $500 \mathrm{ml}$ chyle was drained from the pericardial space. The heart and pericardium were otherwise normal; the thoracic duct could not be identified in the mediastinum but two small structures thought to be lymphatic channels were sutured. No other communication was seen between the lymphatics and pericardial cavity. Subtotal pericardiectomy with abrasion of remaining posterior surfaces was performed.

The postoperative course was uneventful. Two months later he was asymptomatic with normal physical signs. Chest $x$-ray film showed a normal 


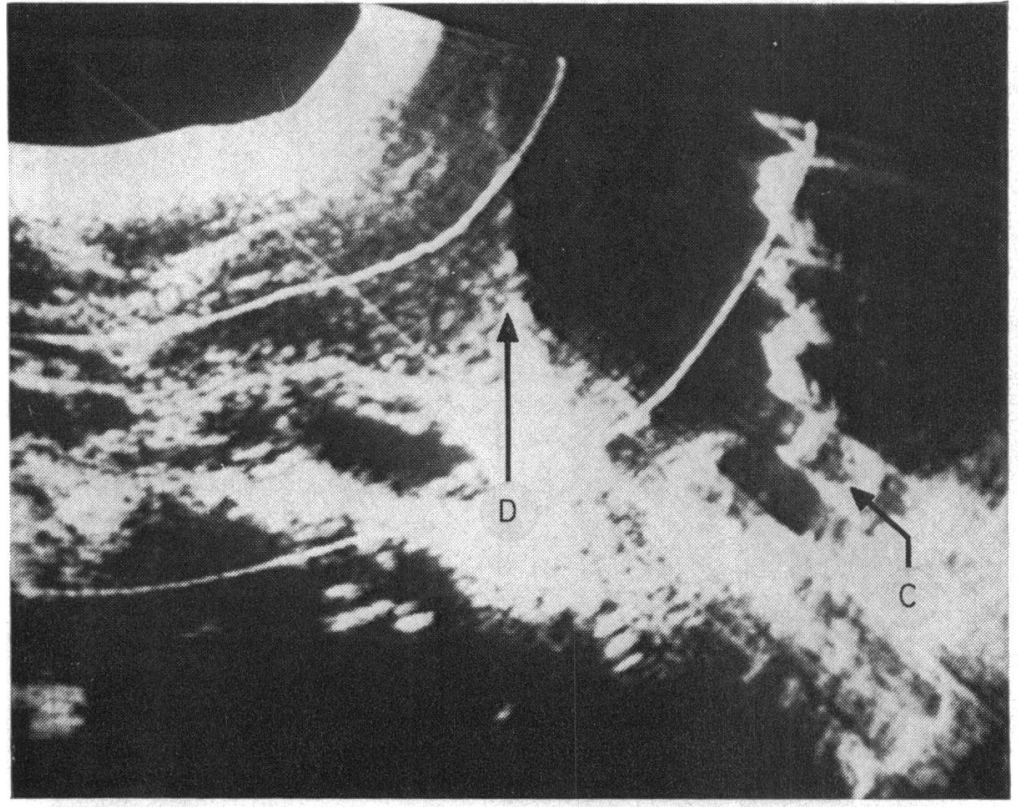

Fig. 3 Contact grey scale $B$-scan ultrasound image showing abnormal separation of diaphragm (D) from the moving lower cardiac border $(C)$ by an echofree space. The image is approximately sagittal, with the patient supine (head to the right).
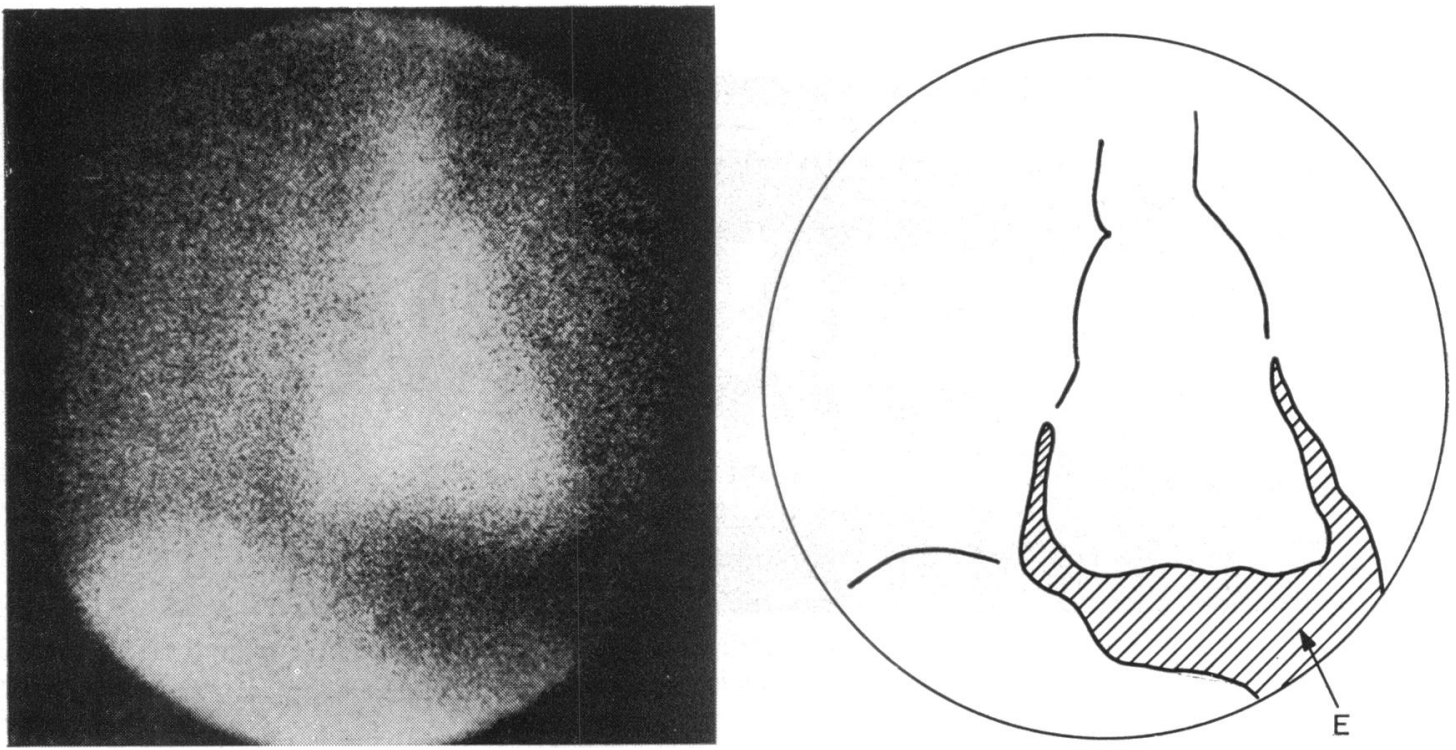

Fig. 4 Thoracic blood pool scan showing abnormal 'halo' surrounding the cardiac silhouette. It is a U-shaped area of decreased radioactivity caused by absorption of the background radiation by the pericardial fluid. Note the area separating the left hemidiaphragm from the base of the heart representing the inferior localisation of the chylous effusion $(E)$.

cardiac silhouette (cardiothoracic ratio 0.40 ) with no evidence of reaccumulation of fluid. The electrocardiogram showed slight ST segment and $\mathrm{T}$ wave changes consistent with pericardiectomy and a change of mean frontal $Q R S$ axis to $+60^{\circ}$. A repeat of the $B$ mode and $M$ mode echocardiograms and thoracic isotope blood pool scan confirmed the absence of pericardial fluid. 
Table Composition of fluid obtained at pericardiocentesis

\begin{tabular}{lll}
\hline Appearance & Milky yellow & \\
Osmolarity & $288 \mathrm{mmol} / 1$ & \\
Cholesterol & $2.4 \mathrm{mmol} / 1$ & $(92.3 \mathrm{mg} / 100 \mathrm{ml})$ \\
Triglycerides & $15.0 \mathrm{mmol} / 1$ & $(1363.6 \mathrm{mg} / 100 \mathrm{ml})$ \\
Protein & $96 \mathrm{~g} / 1$ & \\
Glucose & $4.9 \mathrm{mmol} / 1$ & $(87.5 \mathrm{mg} / 100 \mathrm{ml})$ \\
Sodium & $140 \mathrm{mmol} / 1$ & \\
Potassium & $3.9 \mathrm{mmol} / 1$ \\
Microscopy & Very cellular deposit composed \\
& almost entirely of mature lymphocytes \\
\hline
\end{tabular}

\section{Discussion}

Isolated primary chylopericardium is a rare condition and aetiology remains obscure in most cases. The significance of the previous back injury in our patient is unknown. Only Naef (1956) previously mentioned external injury as a possible cause.

An asymptomatic presentation is not unusual. Of the 22 patients reviewed by Dunn (1975), 8 were asymptomatic when first seen though only 2 were thought to be entirely normal on physical examination.

Diagnosis is by recognition of the presence of a pericardial effusion and demonstration of its chylous nature by pericardiocentesis. In our case the initial diagnosis presented some difficulties and a number of noninvasive techniques were used to overcome them.

Contact grey scale B-scan ultrasound scanning was useful in defining the fluid and localising the site for aspiration. It suggested a cystic cavity lying almost entirely below the heart, even with the patient supine. This inferior localisation of the chyle was possibly related to the chronicity of the effusion and to the narrow anteroposterior chest diameter. $M$ mode echocardiography was initially unhelpful when performed from the usual transducer position in the fourth left intercostal space but it was subsequently successful in showing pericardial fluid when performed from a more inferior position. More inferior transducer positions should, therefore, be routine when standard echocardiographic techniques fail to show a suspected pericardial effusion. Further evidence for the pericardial site of the fluid was obtained by thoracic isotope blood pool scanning after injection of $99 \mathrm{~m}$-Technetium labelled albumin which showed the 'halo' sign typical of pericardial effusion. These techniques proved particularly useful in confirming the absence of fluid after operation.

An interesting and possibly unique radiographic feature was the visualisation of the cardiac border within the outline of the pericardial sac on plain chest radiography. This could be the result of the high fat content and relative transradiancy of chyle compared with heart and blood. It may be a useful early diagnostic clue to the chylous nature of the pericardial fluid.

Surgery was undertaken because the chylous effusion reaccumulated rapidly after pericardiocentesis and it was felt that associated operable tumours such as lymphangiomas and hygromas could not otherwise be definitely excluded.

It is most important that, if it can be identified, the thoracic duct be ligated at operation as all 17 previously reported cases eventually treated with thoracic duct ligation and partial pericardiectomy have survived, with no reaccumulation of fluid. Of 6 previously reported patients who have been treated with partial pericardiectomy alone, 3 required reoperation (with thoracic duct ligation) because of reaccumulation of fluid, 2 died before operation, and 1 refused reoperation. In our case the thoracic duct could not be identified with certainty, but 2 small lymphatic channels were suture ligated and partial pericardiectomy was carried out with abrasion of the remaining surfaces in order to seal off any remaining sites of lymphatic communication. Therefore, if the thoracic duct cannot be identified it seems advisable to seal all lymphatic channels which can be found.

Previous case reports indicated that absence of reaccumulation of chyle after operation was associated with an excellent long term prognosis and this is, therefore, expected in our case.

We thank Dr R. W. Emanuel for advice in the preparation of this report on his patient and Mr M. F. Sturridge (Department of Cardiothoracic Surgery, The Middlesex Hospital) for advice on surgical management and preparation of this report.

We acknowledge the assistance of Dr P. J. Ell (Department of Nuclear Medicine, The Middlesex Hospital) and Dr W. Lees (Department of Clinical Measurement, The Middlesex Hospital).

\section{References}

Dunn, R. P. (1975). Primary chylopericardium: a review of the literature and an illustrated case. American Heart Fournal, 89, 369-377.

Naef, A. P. (1956). Primary chylopericardium and its surgical treatment. Diseases of the Chest, 30, 160-167.

Staab, E. V., and Patton, R. D. (1973). Nuclear medicine studies in patients with pericardial effusion. Seminars in Nuclear Medicine, 3, 191-201.

Requests for reprints to Dr P. J. Ross, Department of Cardiology, The Middlesex Hospital, Mortimer Street, London W1N 8AA. 